\title{
Systematic Brain Data Analysis: A Review
}

\author{
Hamid Faisal \\ M.Tech. Student \\ G.H.R.A.E.T. \\ Nagpur
}

\author{
Shruti Kolte \\ Asst. Professor \\ G.H.R.A.E.T. \\ Nagpur
}

\author{
Vikrant Chole \\ Asst. Professor \\ G.H.R.A.E.T. \\ Nagpur
}

\begin{abstract}
The Brain Data Analysis and evaluation is a challenging task. Focusing towards the human-thinking-centric studies characteristics, brain informatics (BI) emphasizes a systematic methodology with key issue of systematic brain data analysis. The brain data analytical methods are very rare and thus brain data research and implementation is more challenging. This paper reviews the existing research and methodologies developed so far, in the area of Brain Informatics, Web Intelligence, Learning Schemes, Analytical methods and relevant aspects of these along with their limitations. More data and analytical methods need to be added in the existing $\mathrm{BI}$ approaches. Also, there is high need to embed these into a mature computing environment.
\end{abstract}

\section{Keywords}

Brain Informatics (BI), ontologies, domain driven data mining, process planning, Human Information Processing System (HIPS)

\section{INTRODUCTION}

Brain Informatics (BI) is the recent multidisciplinary and interdisciplinary field focusing towards the study of mechanisms underlying the human information processing system (HIPS). BI emphasizes a systematic approach to study human-thinking-centric information processing mechanism from both macro and micro points of view by cooperatively using experimental, theoretical, cognitive neuroscience, and advanced information technologies (e.g., Web intelligence and computational intelligence). Such a systematic approach is generalized as a BI methodology, which is having systematic brain data analysis as a key issue. The systematic BI methodology resulted in the brain big data, for example various raw brain data, data-related information, found domain knowledge related to human intelligence, extracted data features, and so forth. The Data Sources for this field are obtained including but not limited to by the use of powerful equipment like electroencephalogram (EEG), functional magnetic resonance imaging (fMRI), positron emission tomography (PET), eye-tracking, and different wearable, active, ubiquitous,micro and nano devices, as well as the psychological experiments. These various datasets of brain related studies, experiments, ERP/EEG related data, etc. are applied in the projects as input datasets and the various level of processing's performed over them, like evaluation of analytical agents over those data agents. The data agents refer to any data instances of any type. Thus the process planning is performed over those. As a domain driven data mining, Brain Informatics target the discovery of thinking related brain structures and mechanisms rather than production of automatic algorithms and tools.

The Remainder of this paper is organized as follows:

Section II discusses background and prerequisites relating to the subject area. Furthermore, Section III discusses the related work done in past and the studies taken place in the previous papers of research. Finally, Section IV gives concluding remarks about the study we have performed in this paper.

\section{BACKGROUND}

\subsection{The Data Brain Model}

The Data-Brain [1][2][20] is a domain-driven conceptual model for brain data, which represents multi-aspect relationships among numerous human brain data sources, with respect to all major capabilities and aspects of HIPS, for systematic investigation and understanding of human intelligence. It is neither a digital brain which models brain structures by digital and visual technologies nor a logical brain which models brain functions for the simulation and the development of new IT technologies.

The [20] have shown supporting capabilities, features, and construction of such Data-Brain model. This Data Brain is claimed to support various methods for data analysis, simulations, visualizations as well as being analogous to knowledge and models. For supporting the systematic investigation and understanding of human intelligence in BI, the Data-Brain models heterogeneous brain data and multiaspect relationships among them at the conceptual level to integrate key data, information and knowledge for the constructions of various research supporting systems which can form a BI data cycle system to carry out the systematic BI methodology and support the whole BI research processes.

In [2], the Data-Brain modeling has been shown as a core issue of BI study, which is with multiple conceptual views and its own four dimensions corresponding to the four aspects of systematic BI methodology. Such a Data-Brain can be constructed by a BI methodology based ontological modeling approach. Two realistic use cases shown about how the DataBrain can be used for various data requests which are coming from different aspects of a systematic BI study. As the core of BI data cycle system, the Data-Brain represents a radically new ways of storing and sharing data and knowledge, as well as enables high speed, distributed, large-scale, multi-aspect analysis and computation on the Wisdom Web and knowledge grids.

Conceptual modeling approaches can be divided into two types: conceptual data modeling and ontology modeling. Although both ontologies and data models are partial accounts of conceptualizations [14] and share many common features, they do have some differences. Fonseca et al. defined two criteria to differentiate ontologies from conceptual data models: the objectives of modeling and objects to model.

Data brain provides a formal conceptual framework to integrate various data information and knowledge for systematic brain informatics studies; especially brain data analysis. On brain informatics methodologies base, the [1] proposed new approach towards data-brain model driven systematic brain data analysis approach using a multi-agent system named Global Learning System (GLS) for brain 
informatics (GLS-BI). Such a Development is based on the below observations:

Firstly, Development of data brain driven approach was necessary for systematic data brain analysis. Studies of human thinking centric cognitive functions are complex and in all incorporate with multiple interrelated functions with respect to activated brain areas for a given task and neural structure and biological processes related to the activated areas. Following the characteristics of thinking centric studies, BI needs to implement the systematic brain data analysis for the purpose of understanding human thinking centric information processing mechanism rather than understanding of brain data by one or several effective analytical method; by integrating closely related data sources and various analytical methods. Such Integration is domain knowledge driven and is difficult to be implemented because of explosive growth of both data and analytical methods. Thus, it was very important to develop new technologies and tools to integrate both data and analytical methods for systematic brain data analysis.

Secondly, it is possible to develop the same. By following the systematic investigation and the experimental design of BI methodology, human brain data obtained in systematic studies are interrelated and can be utilized for multiple purposes.

In summary that the [1] systematic brain data analysis can be regarded domain driven data mining [5] in brain science which course issue of integrating various brain data and analytical method for in-depth understanding of human thinking. for understanding it $\mathrm{i}$ need to develop a data brain driven approach systematic bacteriology also make it happen to realize search data plane application.

\subsection{Systematic Brain Data Analysis}

Systematic Brain Data Analysis [1] [5] is an important issue of BI methodology. As a domain-driven data mining, it targets not the production of automatic algorithms and tools but the discovery of thinking-related brain structures and mechanisms.

Previous study [2], proposed multiaspect analytical approach to implement systematic print data analysis. It can be generalized as following four steps:

1) Purpose definition: Investigators choose a thinking-related cognitive Function (e.g., computation) as the objective cognitive Function, i.e., the analytical object.

2) Typical analysis: Aiming at the objective cognitive function, investigators respectively analyze the brain data obtained by different experiments and understand them based on the mining results, i.e., obtained spatiotemporal features.

3) Exploratory analysis: Aiming at the objective cognitive function and "similar" cognitive functions, investigators synthetically analyze the corresponding experimental data to find key spatiotemporal features which are important in the information processing course of objective cognitive function.

4) Specific analysis: Focusing on the obtained key spatiotemporal features, investigators contrastively analyze the objective cognitive function and "functionally related" cognitive functions to understand these spatiotemporal features in depth.

The said approach is 'holistic' approach which doesn't aim at a or several data sources but at a thinking related cognitive function and emphasizes integrating various data and analytical methods to understand the objective cognition function in depth by a gradual deepening process. Such an approach is domain driven. The domain knowledge, information related to data and analytical methods, and investigators individual suppositions (for example how to identify 'functionally related' cognitive functions) need to be synthetically utilized.

\subsection{KDD Process Planning}

The key issue of multi aspect data analysis is how to find and $\mathrm{i}$ integrate needed it and analytical method according to different analytical purpose the genius provide practical approach installing this issue. The models are formed on knowledge discovery and data mining KDD process basis as an organizer city of autonomic knowledge discovery agent for dynamically organizing and managing the KDD process based on ontology of KDD agents.

\section{LITERATURE REVIEW}

In [1], a multiagent system, named Global Learning Scheme for BI (GLS-BI) has been proposed that is claimed to perform the data-brain driven process planning. They proposed that it could perform the KDD process planning to integrate the needed data and analytical methods and create various mining workflows for guiding the multiaspect brain data analysis. This approach adopted the agent-Based Web Service workflow model. Depending upon GLS methodology, [1] proposed multi agent system named GLS-BI. I is claimed to perform KDD process planning to integrate needed data and analytical method and thus create various mining workflows for the sake of guiding multiaspect brain data analysis. The GLS-BI adopts agent base web service workflow model. Its main components include formal knowledge base, open agent society and a process planning engine of KDD. Formal knowledge base of GLS-BI consists of two subcomponents viz. data of brain and BI provenances.

Data brain is a conceptual data model that represents functional relationship among multiple human brain data sources with respect to always respects and capabilities of human information processing system (HIPS); for the sake of systematic investigation and understanding of human intelligence [2].

The previous studies [1][19], claimed to constructed a prototype of data brain using ontology web language description logics (OWL-DL) [18] which is induction centric and describes human induction related cognitive functions. It includes 4 dimensions which are responding to 4 issues of systematic methodologies respectively. Different from common provenances models such as open provenance model [14], the OWL-DL-based prototype uses the special concepts $\&$ relations of BI present various elements of variance models, Special provenance model of BI.

The GLS-BI [1] has been claimed to be advantageous in terms of two aspects. Firstly, the standardizing of the analytical processes by integrating domain knowledge metadata and investigators' individual suppositions (custom rules) and thus generating mining workflows and forming three step analytical process. Secondly the improvement of the data utilization by the data-brain driven process planning.

However, the paper specified to obtain only some preliminary results. Furthermore, the wrapping of more data and analytical methods are thrown as a future work. Also, embedding it into a mature distributed computing environment is kept for the future work as well.

The [2], explained the Data-Brain, a conceptual model of brain data and How to construct it. Data-Brain explicitly 
represents numerous relationships between numerous human brain data sources, with respect to all major capabilities and aspects of human information processing systems (HIPS). As per this study, Conceptual modeling as explained, divided into two categories viz. conceptual data modeling, and the ontology modeling. Even though they are sharing many common, features, still, they have some differences as well. The BI methodology based modeling Terms Gathering, Construction of Function Dimension depending on Systematic Investigations, and constructing experiment dimension, Constructing Data Dimension depending on Systematic Data Management, Constructing Analysis Dimension, and then Extraction of Conceptual views from function dimension and constructing the Relations among various dimensions for BI provenances were the steps for evolution of data brain.

The [3], shown that the systematic BI methodology resulted in the brain big data, including various raw brain data, datarelated information, extracted data features, found domain knowledge related to human intelligence, and so forth. They discussed research issues and challenges from three aspects of BI studies that are worth deserving closer attention viz. systematic investigations for complex brain science problems, new information technologies for supporting systematic brain science studies, and BI studies depending upon needs of Web intelligence research. Human Intelligence is shown as one of the latest research aspects. Core three aspects and issued of BI research discussed. Both Web and the Brain are shown symmetric as both are complex networks with big data. The interrelations between Brain Informatics, Web Intelligence and Human Information Processing System, Clinical Researches and other Experiments are well specified in this study.

In [4], the notion of Wisdom Web of Things (W2T) is proposed in order to address the urging research issue to realize the organic combination and symmetrical mutualism among humans, computers, and things in the hyper world, that consists of social world, physical world and the information world (cyber world). Integrating the existing studies of intelligent information technologies, they proposed theW2T as a holistic intelligence methodology in the hyper world. AW2T data cycle system is said designed to drive the cycle, namely "from things to data, information, knowledge, wisdom, services, humans, and then back to things" for realizing the W2T. The comparison between World Wide Web and the W2T is illustrated. The cycle of the hyper world is shown as between Humans and the Things as Data to Information, Information to Knowledge, Knowledge to Wisdom, Wisdom to Services, Services to Humans and Humans to the Things. Also, the Hyper World is shown parallel between the Social World and the Cyber World.

In [17], outlined the key research topics of BI are shows as thinking centric investigation of HIPS including human reasoning mechanism and the human learning mechanism, perception centric investigation of HIPS including human multi-perceptive mechanism and the auditory, visual, tactile information processing, and modeling human brain information processing mechanism including neuromechanisms of HIPS, mathematical models of HIPS, cognitive and computational mechanisms of HIPS, and the Information Technologies for management and use of human brain data The Paper specifies how the BI meets WI in Principle, how BI meets WI meets BI in fundamental research and finally, impending 'WI meets BI' research. Web intelligence, according to this study is becoming a central area that revolutionizes artificial intelligence and information technologies to achieve human-level Web intelligence.

The [21], specified self-adaptiveness and the harmonious intelligence in the hyper world. The Self adaptive technologies or procedure in W2T is shown there. It refers the adaptive requirement description language as two parts viz. wisdom-service description files and the self adaptive wisdom-service scheme definition files. All these files are described in XML form. The Reasoning and planning in W2T is specified in this paper. The extended Heuristic Rete Algorithm is used to achieve forward reasoning for this. The Heuristic Task Network (HTN) is also described to achieve backward planning.

Table 1 : A Comparative Summary of Reviews of Literature

\begin{tabular}{|c|l|l|l|}
\hline $\begin{array}{c}\text { Sr. } \\
\text { No. }\end{array}$ & \multicolumn{1}{|c|}{ Paper } & \multicolumn{1}{c|}{ Major Findings } & \multicolumn{1}{c|}{ Limitations/Insufficiencies/Else } \\
\hline 1 & $\begin{array}{l}\text { Toward Data-Brain } \\
\text { Driven Systematic } \\
\text { Brain Data Analysis }\end{array}$ & $\begin{array}{l}\text { Global Learning Scheme for BI } \\
\text { proposed. Multi-step process planning is } \\
\text { shown. Analysis Agent Discovery } \\
\text { Algorithm specified. }\end{array}$ & $\begin{array}{l}\text { Specified to obtain only some preliminary results. } \\
\text { Wrapping of more data and analytical methods are } \\
\text { thrown as a future work. Embedding it into a mature } \\
\text { distributed computing environment kept for future. }\end{array}$ \\
\hline 2 & $\begin{array}{l}\text { Constructing a New- } \\
\text { style Conceptual } \\
\text { Model of Brain Data } \\
\text { for Systematic Brain } \\
\text { Informatics }\end{array}$ & $\begin{array}{l}\text { Theory of construction od Data-Brain } \\
\text { has been specified. Algorithms for } \\
\text { Conceptual view Extraction and getting } \\
\text { TDR (Traversal Direct Result) shown. }\end{array}$ & $\begin{array}{l}\text { It simply specifies the new way of radically storing and } \\
\text { sharing of data and knowledge. }\end{array}$ \\
\hline 3 & $\begin{array}{l}\text { Research Issues and } \\
\text { Informatics Towards } \\
\text { Computing \& } \\
\text { Intelligence in the Big } \\
\text { Data Era }\end{array}$ & $\begin{array}{l}\text { Human Intelligence is shown as one of } \\
\text { the latest research aspects. Core Three } \\
\text { aspects and issued of BI research } \\
\text { discussed. Both Web and the Brain are } \\
\text { shown symmetric as both are complex } \\
\text { networks with big data. }\end{array}$ & $\begin{array}{l}\text { The Aspects and issues of current and expected research } \\
\text { specified. }\end{array}$ \\
\hline
\end{tabular}




\begin{tabular}{|l|l|l|l|}
\hline 4 & $\begin{array}{l}\text { Research challenges } \\
\text { and perspectives on } \\
\text { Wisdom Web of } \\
\text { Things (W2T) }\end{array}$ & $\begin{array}{l}\text { AW2T data cycle system is said } \\
\text { designed to drive the cycle, namely } \\
\text { "from things to data, information, } \\
\text { knowledge, wisdom, services, humans, } \\
\text { and then back to things" for realizing } \\
\text { the W2T. The comparison between } \\
\text { World Wide Web and the W2T is } \\
\text { illustrated. }\end{array}$ & $\begin{array}{l}\text { Research challenges and perspectives on Wisdom Web of } \\
\text { Things is specified. They need to be addressed or coped } \\
\text { with. }\end{array}$ \\
\hline 5 & $\begin{array}{l}\text { Web Intelligence } \\
\text { meets brain } \\
\text { informatics }\end{array}$ & $\begin{array}{l}\text { The paper describes BI and WI as } \\
\text { complementary and supportive to each } \\
\text { other. }\end{array}$ & $\begin{array}{l}\text { The specific functional methodology is not shown in the } \\
\text { paper. }\end{array}$ \\
\hline $\begin{array}{l}\text { Adaptive support } \\
\text { framework for } \\
\text { wisdom web of things }\end{array}$ & $\begin{array}{l}\text { The Reasoning and planning in W2T is } \\
\text { specified in this paper. The extended } \\
\text { Heuristic Rete Algorithm is used to } \\
\text { achieve forward reasoning for this. The } \\
\text { Heuristic Task Network (HTN) is also } \\
\text { described to achieve backward } \\
\text { planning. }\end{array}$ & $\begin{array}{l}\text { Paper furthermore says to have many challenges to } \\
\text { achieve harmonious intelligence. Many research issues } \\
\text { specified needs to be addressed to conduct further studies } \\
\text { in wisdom service model description language, reasoning } \\
\text { and planning techniques and learning algorithms. }\end{array}$ \\
\hline
\end{tabular}

\section{CONCLUSION}

The Brain Informatics is the revolutionary trend that is going to change the complete face of Computational Intelligence, Human Level Processing, and Web Intelligence. Traversing through data, information, and knowledge; the computed intelligence is seen expected to reach wisdom of things (Wisdom Web of Things). The Data-Brain, a conceptual data model for modeling brain data, tries helping the systematic brain data analysis. Since this Area is very new, only some research, studies or works are carried out and only few preliminary results are obtained so far, as claimed.

The Wrapping of more data and analytical methods into the proposed models, the GLS-BI is needed. The challenges exist in analysis agent discovery, implementation of humanthinking centric cognitive functionality, embedding it in a mature web computing environment, and estimation of agent interactive learning scheme, and much more. Thus, there is plenty of future scope in the area of Brain Informatics and the systematic Brain Data Analysis as well as its utilization upon it.

\section{REFERENCES}

[1] Jianhui Chen and Ning Zhong, "Toward the Data-Brain Driven Systematic Brain Data Analysis", IEEE TRANS. ON SYSTEMS, MAN, AND CYBERNETICS: SYSTEMS, VOL. 43, NO.1, JAN-2013

[2] Ning Zhong and Jianhui Chen, "Constructing a Newstyle Conceptual Model of Brain Data for Systematic Brain Informatics",

[3] Ning Zhong, "Research Issues and Challenges on Brain Informatics Towards Computing \& Intelligence in the Big Data Era”, IEEE TRANS. ON KNOWLEDGE AND DATA ENGINEERING

[4] Ning Zhong, Jian Hua, et al. "Research challenges and perspectives on Wisdom Web of Things (W2T)", Springer Science+Business Media, LLC 2010 online publication, (26 Nov 2010, J Supercomput DOI $10.1007 / \mathrm{s} 11227-010-0518-8)$

[5] Ning Zhong, "WI Based Multi-Aspect Data Analysis in a Brain-Informatics Portal” , Automonous Intelligent Systems: Agents and Data Mining, June 2007
[6] Jianhui Chen, et. al., "Data-Brain Modeling Based on Brain Informatics Methodology", Web Intelligence and Intelligent Agent Technology, 2008. WI-IAT '08. IEEE/WIC/ACM International Conference on (Volume:1)

[7] Ning Zhong and Shinichi Motomura, "Agent-Enriched Data Mining: A Case Study in Brain Informatics" , In proc. of Agents And data Mining, IEEE INTELLIGENT SYSTEMS, www.computer.org/intelligent

[8] Jianhua Ma, et. al., "Cyber-Individual Meets Brain Informatics" , Brain Informatics, 1541-1672/11, IEEE INTELLIGENT SYSTEMS , SEP/Oct 2011 www.computer.org/intelligent

[9] Wang, Yingxu, et. al., "Contemporary Cybernetics and Its Facets of Cognitive Informatics and Computational Intelligence", Systems, Man, and Cybernetics, Part B: Cybernetics, IEEE Transactions on(Volume:39, Issue:4 )

[10] Ning Zhong et. al. , "Brain Informatics" , Intelligent Systems, IEEE (Volume:26, Issue: 5 )

[11] Yulin Qin, "Various Levels from Brain Informatics to Web Intelligence", Web Intelligence and Intelligent Agent Technologies, 2009. WI-IAT '09. IEEE/WIC/ACM International Joint Conferences on (Volume:1 )

[12] Jianhui Chen , "Multi-Task Learning via Structured Regularization: Formulations, Algorithms, and Applications", A Dissertation Presented in Partial Fulfillment of the Requirements for the Degree Doctor of Philosophy, Approved August 2011 by the Graduate Supervisory Committee , ARIZONA STATE UNIVERSITY

[13] M. Jarrar, J. Demey, and R. Meersman, "On Using Conceptual Data Modeling for Ontology Engineering," Journal of Data Semantics, vol. 1, no. 1, pp. 185-207, 2003.

[14] M. Uschold and M. Gruninger, "Ontologies principles, methods and applications", Knowledge Engineering Review, vol. 11, no. 2, pp. 93-155, 1996. 
[15] F. Fonseca and J. Martin, "Learning the Differences Between Ontologies and Conceptual Schemes through Ontology-driven Information Systems," Journal of the Association for Information Systems - Special Issue on Ontologies in the Context of IS, vol. 8, no. 2, pp. 129$142,2007$.

[16] L. B. Cao and C. Q. Zhang, "The evolution of KDD: Towards domain driven data mining," Int. J. Pattern Recognit. Artif. Intell., vol. 21, no. 4, pp.677-692, 2007.

[17] N. Zhong, J. Liu, Y. Yao, J. Wu, S. Lu, Y. Qin, K. Li, and B. Wah, "Web Intelligence meets brain informatics," in Proc. WImBI, 2007, pp. 1-31.

[18] D. L. McGuinness and F. V. Harmelen, OWL Web Ontology Language Overview, Feb.

$\begin{array}{lcr}\begin{array}{l}\text { 2004, WorldWideWeb } \\ \text { recommendation. }\end{array} & \begin{array}{c}\text { Consortium } \\ \text { [Online]. }\end{array} & \text { (W3C) } \\ \text { Available: }\end{array}$
http://www.w3.org/TR/owl-features/

[19] J. H. Chen and N. Zhong, "Data-brain modeling for systematic brain informatics," in Proc. Int. Conf. BI, 2009, pp. 182-193.

[20] Jianhui Chen , Ning Zhong Data-Brain Modeling Based on Brain Informatics Methodology, 2008 IEEE/WIC/ACM International Conference on Web Intelligence and Intelligent Agent Technology.

[21] Yang Gao, Mufeng Lin, Ruili Wang, "Adaptive support framework for wisdom web of things", World Wide Web (2013) 16:379-398, DOI 10.1007/s11280-012-0183-3. 